\title{
Evaluation of a Pulsed Xenon Ultraviolet Disinfection System for Reduction of Healthcare-Associated Pathogens in Hospital Rooms
}

\author{
Michelle M. Nerandzic, BS; ${ }^{1}$ Priyaleela Thota, MD; ${ }^{2}$ Thriveen Sankar C., MBA; ${ }^{2}$ Annette Jencson, MT, CIC; ${ }^{1}$ \\ Jennifer L. Cadnum, $\mathrm{BS}^{2}{ }^{2}$ Amy J. Ray, MD; ${ }^{2,3}$ Robert A. Salata, MD; ${ }^{2,3}$ Richard R. Watkins, $\mathrm{MD} ;{ }^{4}$ Curtis J. Donskey, $\mathrm{MD}^{2,3,5}$
}

ов јестіле. To determine the effectiveness of a pulsed xenon ultraviolet (PX-UV) disinfection device for reduction in recovery of healthcareassociated pathogens.

SETTING. Two acute-care hospitals.

MEthods. We examined the effectiveness of PX-UV for killing of Clostridium difficile spores, methicillin-resistant Staphylococcus aureus (MRSA), and vancomycin-resistant Enterococcus (VRE) on glass carriers and evaluated the impact of pathogen concentration, distance from the device, organic load, and shading from the direct field of radiation on killing efficacy. We compared the effectiveness of PX-UV and ultraviolet-C (UV-C) irradiation, each delivered for 10 minutes at 4 feet. In hospital rooms, the frequency of native pathogen contamination on high-touch surfaces was assessed before and after 10 minutes of PX-UV irradiation.

RESUlts. On carriers, irradiation delivered for 10 minutes at 4 feet from the PX-UV device reduced recovery of $C$. difficile spores, MRSA, and VRE by $0.55 \pm 0.34,1.85 \pm 0.49$, and $0.6 \pm 0.25 \log _{10}$ colony-forming units $(\mathrm{CFU}) / \mathrm{cm}^{2}$, respectively. Increasing distance from the PX-UV device dramatically reduced killing efficacy, whereas pathogen concentration, organic load, and shading did not. Continuous UV-C achieved significantly greater $\log _{10} \mathrm{CFU}$ reductions than PX-UV irradiation on glass carriers. On frequently touched surfaces, PX-UV significantly reduced the frequency of positive $C$. difficile, VRE, and MRSA culture results.

CONCLUSions. The PX-UV device reduced recovery of MRSA, C. difficile, and VRE on glass carriers and on frequently touched surfaces in hospital rooms with a 10-minute UV exposure time. PX-UV was not more effective than continuous UV-C in reducing pathogen recovery on glass slides, suggesting that both forms of UV have some effectiveness at relatively short exposure times.

Infect Control Hosp Epidemiol 2015;36(2):192-197

Automated room disinfection technologies are increasingly being used as an adjunct to standard cleaning and disinfection in healthcare facilities. Ultraviolet (UV) radiation devices have been most widely adopted owing to the efficiency and welldocumented efficacy of UV irradiation. ${ }^{1-7}$ Several UV room disinfection devices are now being marketed. Most of these devices use low pressure mercury gas bulbs, but recently pulsed xenon flash bulbs have also been incorporated into disinfection systems. UV radiation has peak germicidal effectiveness in the wavelength range from 240 to $280 \mathrm{~nm}^{1-7}$ Mercury gas bulbs primarily emit UV-C at $254 \mathrm{~nm}$, whereas xenon gas bulbs produce a broad spectrum of radiation that encompasses the UV (100-280 nm) and visible (380-700 nm) spectra. ${ }^{8-12}$ The UV-C radiation emitted by low pressure mercury bulbs is delivered in a continuous stream that gradually accumulates to lethal doses depending on duration of exposure and distance from the primary field of radiation. ${ }^{1-7}$ The broad-range UV delivered by xenon bulbs is emitted in short, high-intensity pulses, possibly requiring a shorter duration of exposure to achieve lethal doses. ${ }^{8-12}$

Given the increasing use of UV devices and variations in recommended cycle times, there is a need for evaluations of the real-world performance and comparative effectiveness of different devices. We previously demonstrated that a mobile, automated room disinfection device that utilizes mercury bulbs for emitting UV-C radiation is effective for reducing the frequency of positive methicillin-resistant Staphylococcus aureus (MRSA), vancomycin-resistant Enterococcus (VRE), and Clostridium difficile culture results on high-touch surfaces in hospital rooms (Tru-D Rapid Room Disinfection device; Lumalier). ${ }^{13}$ For disinfection of vegetative bacteria and $C$. difficile spores, the manufacturer recommends cycles in standard

Affiliations: 1. Research Service, Louis Stokes Cleveland Veterans Affairs Medical Center, Cleveland, Ohio; 2. Case Western Reserve University School of Medicine, Cleveland, Ohio; 3. Department of Medicine, University Hospitals Case Medical Center, Cleveland, Ohio; 4. Akron General Medical Center, Akron, Ohio; 5. Geriatric Research, Education and Clinical Center, Cleveland Veterans Affairs Medical Center, Cleveland, Ohio.

Received July 11, 2014; accepted October 14, 2014; electronically published January 5, 2015

(c) 2015 by The Society for Healthcare Epidemiology of America. All rights reserved. 0899-823X/2015/3602-0010. DOI: 10.1017/ice.2014.36 
hospital rooms of approximately 15 and 45 minutes, respectively. Here we examined the effectiveness of a mobile, automated pulsed xenon ultraviolet (PX-UV) device (Xenex; Xenex Disinfection Services) at a substantially shorter disinfection cycle (10 minutes, as suggested by manufacturer). The efficacy of the device was assessed for killing of MRSA, VRE, and C. difficile spores on carriers placed in hospital rooms and for reducing naturally occurring contamination on high-touch surfaces in hospital rooms.

\section{METHODS}

\section{C. difficile, MRSA, and VRE Strains}

Two clinical isolates each of $C$. difficile, MRSA, and VRE were studied. The MRSA strains were a pulsed-field gel electrophoresis type USA300 and USA800. The VRE strains were a VanA-type isolate (C37) and a VanB-type isolate (C68). The C. difficile strains were VA 17, a restriction endonuclease analysis type BI strain, and VA 11, a restriction endonuclease analysis type J strain.

\section{Preparation of C. difficile Spores}

Spores were prepared as previously described. ${ }^{14}$ Spores were stored at $4^{\circ} \mathrm{C}$ in sterile distilled water until use. Prior to testing, spore preps were confirmed by phase contrast microscopy and malachite green staining to be at least $99 \%$ dormant, bright-phase spores.

\section{Microbiology}

VRE, MRSA, and C. difficile were cultured on selective media as previously described. ${ }^{13,15}$ For specimens collected with sterile, premoistened swabs, the swabs were applied directly to the surface of the appropriate selective agar. To detect lower levels of $C$. difficile spores, $10 \mathrm{~mL}$ of pre-reduced cycloserinecefoxitin-brucella broth containing $0.1 \%$ taurocholic acid and lysozyme $5 \mathrm{mg} / \mathrm{mL}$ (CDBB) was poured into a sterile culture tube containing specimens collected with sterile gauze pads. ${ }^{15}$ Positive broth cultures were subcultured onto selective agar for identification of $C$. difficile. To quantify total heterotrophic bacteria, swabs were plated on trypticase soy agar containing $5 \%$ sheep blood and incubated at $37^{\circ} \mathrm{C}$ for 48 hours. VRE and MRSA colonies with unique morphology were subjected to identification and susceptibility testing in accordance with Clinical Laboratories Standards Institute guidelines. ${ }^{16}$ C. difficile was confirmed on the basis of odor and appearance of colonies and by a positive reaction using $C$. difficile latex agglutination (Microgen Bioproducts).

\section{The Pulsed Ultraviolet Disinfection Device}

Figure 1 is a photograph of the PX-UV device (Xenex; Xenex Disinfection Services). The device contains a xenon gas flash bulb that operates at $2 \mathrm{~Hz}$ and emits a broad spectrum of

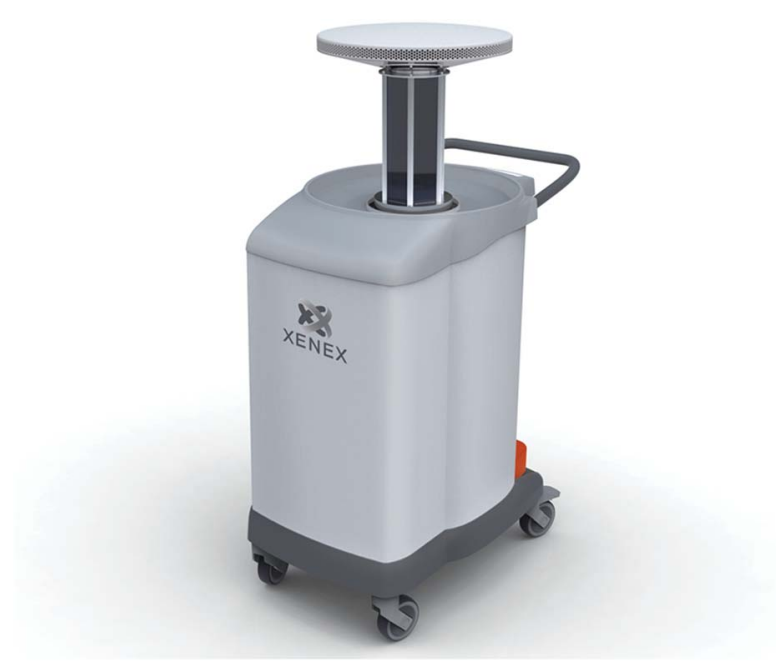

FIGURE 1. Photograph of the Xenex pulsed xenon ultraviolet device.

radiation covering the UV-C spectrum of 200 to $280 \mathrm{~nm}$ as well as the visible light spectrum. The device is designed for manipulation by a single operator and is approximately $1.6 \mathrm{ft}$ wide by $2.3 \mathrm{ft}$ long by $3.3 \mathrm{ft}$ high and weighs 150 pounds. It is operated remotely outside the room and includes motion sensors, which turn off the device if the door is opened. The device is wheeled into a strategic position located near high-touch surfaces in the room and set to irradiate for 5 to 7 minutes as suggested by the manufacturer. Then, the device is wheeled to a second location in the room and run for an additional 5 to 7 minutes. The disinfection process takes approximately 15 to 20 minutes, which includes setup, radiation cycles, and repositioning.

\section{The Impact of Pathogen Concentration and Organic Load on the Efficacy of PX-UV Disinfection on Carriers in Hospital Rooms}

Initial experiments were conducted to determine whether pathogen concentration (ie, colony-forming units [CFU] per $\mathrm{cm}^{2}$ ) or organic load influenced the disinfection efficacy of the PX-UV device. Ten $\mu \mathrm{L}$ aliquots of $C$. difficile spores, MRSA, and VRE suspended in phosphate buffered saline were inoculated onto glass microscope slides and spread to cover a $1-\mathrm{cm}^{2}$ area. For each pathogen, the inoculum applied to the slide was adjusted such that 2 to 5 or more $\log _{10} \mathrm{CFU} / \mathrm{cm}^{2}$ were recovered from the positive control specimens after desiccation. For a subset of samples, the organisms were suspended in $5 \%$ fetal calf serum.

The slides were placed on a table positioned centrally over the bed in a hospital room, 4 feet within the direct field of radiation delivered by the PX-UV device. Baseline slides were left untreated outside of the room (ie, positive controls). The PX-UV device was run for a total of 10 minutes, 5 minutes on the left side of the bed and 5 minutes on the right, as suggested 
by the manufacturer and standard protocol in the facility utilizing the device.

To quantify viable organisms, the slides were submersed in $25 \mathrm{~mL}$ of sterile phosphate buffered saline and vortexed vigorously, and dilutions of the suspensions were plated onto selective media. Following 48 hours of incubation, $\log _{10} \mathrm{CFU}$ reductions were calculated by comparing the $\log _{10} \mathrm{CFU}$ recovered from slides after PX-UV disinfection to untreated controls. All experiments were performed 3 times.

\section{The Impact of Distance on the Efficacy of PX-UV Disinfection on Carriers in Hospital Rooms}

The killing efficacy of the PX-UV device was evaluated at increasing distances from the primary field of radiation. Slides were prepared and processed as described previously. However, the inoculum was altered such that each glass slide yielded $5 \log _{10}$ CFU at baseline. Additionally, slides were placed 6 inches, 4 feet, and 10 feet within the direct field of radiation, and also 4 feet shaded from direct radiation (under bedside table).

\section{Comparison of Pulsed Xenon Versus Continuous Mercury UV for Killing of Pathogens}

We compared the efficacy of PX-UV versus UV-C delivered by mercury bulbs for reduction of pathogens inoculated onto slides in similarly sized hospital rooms. This comparison was performed in separate facilities because the devices were housed in separate hospitals. The experiments were performed in similar rooms with equivalent dimensions, and the experimental samples were placed at the same distances from the UV devices. Slides were prepared as described previously; the inoculum was altered such that each glass slide yielded $5 \log _{10}$ $\mathrm{CFU}$ at baseline. The slides were placed 4 feet from each device within the direct field of radiation. The UV-C was delivered by the Tru-D device (Lumalier); each device was run for a total of 10 minutes. Slides were processed as described previously. The experiments were performed 3 times.

\section{Disinfection of Environmental Surfaces in Hospital Rooms}

The efficacy of the PX-UV device was assessed in rooms $(\sim 10 \times 20$ feet $)$ of discharged patients in a tertiary care facility. In phase 1, the PX-UV device was run in rooms that had not yet been cleaned. In phase 2, the device was run after standard terminal cleaning by environmental services personnel that included use of bleach for high-touch surfaces in all discharge rooms; a subset of the rooms had previously been occupied by patients with $C$. difficile infection. Swabs and gauze pads premoistened with saline were used to collect cultures for MRSA, VRE, C. difficile, and total heterotrophic bacteria from hightouch surfaces (ie, call light, bedside table, telephone, chair, intravenous poles, portable keyboards, and bed rail) before and after use of the PX-UV device for 10 minutes ( 5 minutes on each side of the bed). An approximately $10 \times 10-\mathrm{cm}$ area was cultured before PX-UV disinfection and adjacent areas of the same size were cultured after disinfection. Specimens were cultured and identified as described previously.

\section{Data Analysis}

Data were analyzed using STATA, version 9.0 (StataCorp). Continuous data were analyzed using paired $t$ tests and categorical data were assessed using the Fisher exact test.

\section{RES ULT S}

Figure 2 shows the mean $\log _{10} \mathrm{CFU} / \mathrm{cm}^{2}$ reductions of 2 strains of $C$. difficile, MRSA, and VRE on glass slides after the use of the PX-UV device. There were no significant differences between the $\log _{10} \mathrm{CFU}$ reductions of the 2 strains of each pathogen tested. Therefore, in subsequent experiments, data for the 2 strains was pooled. Pathogen concentration did not have a significant impact on the killing efficacy of the PX-UV device. Irradiation delivered 4 feet from the PX-UV device for 10 minutes reduced $C$. difficile spores by $0.55 \pm 0.34 \log _{10} \mathrm{CFU} /$ $\mathrm{cm}^{2}$, MRSA by $1.85 \pm 0.49 \log _{10} \mathrm{CFU} / \mathrm{cm}^{2}$, and VRE by $0.6 \pm 0.25 \log _{10} \mathrm{CFU} / \mathrm{cm}^{2}$. Organic load (5\% fetal calf serum) did not significantly impact the efficacy of the PX-UV device (data not shown).

As shown in Figure 3, the efficacy of PX-UV decreased as distance from the device increased. For each pathogen, significantly less reduction was achieved at 4 feet versus 6 inches and at 10 feet versus 4 feet $(P<.05$ for each comparison). At 4 feet from the device, shading the organisms from the direct field of radiation did not have a significant impact on efficacy ( $P>.05$ for each comparison). At 10 feet from the device, the $\log _{10} \mathrm{CFU}$ reduction was less than $1 \log _{10} \mathrm{CFU} / \mathrm{cm}^{2}$ for each pathogen.

Figure 4 shows the mean $\log _{10} \mathrm{CFU} / \mathrm{cm}^{2}$ reductions of $C$. difficile, MRSA, and VRE on slides after the use of the UV-C and PX-UV devices for 10 minutes at a distance of 4 feet from the devices. The UV-C device achieved significantly greater $\log _{10} \mathrm{CFU}$ reductions than the PX-UV device $(P<.001$ for each pathogen).

Table 1 provides a summary of the results of 2 phases of PXUV disinfection on high-touch surfaces in hospital rooms. For 16 rooms that were cultured before and after use of PX-UV without cleaning (phase 1), PX-UV resulted in statistically significant reductions in the percentages of sites positive for each of the 3 pathogens, the number of CFU recovered for each pathogen, and the heterotrophic plate counts. For 24 rooms that were cultured before and after standard cleaning plus PX-UV (phase 2), there were also statistically significant reductions in percentages of sites positive for each of the pathogens, the number of CFU recovered, and the heterotrophic plate counts.

\section{DISCUSSION}

We found that the PX-UV device reduced recovery of MRSA, C. difficile, and VRE on carriers and on frequently touched 


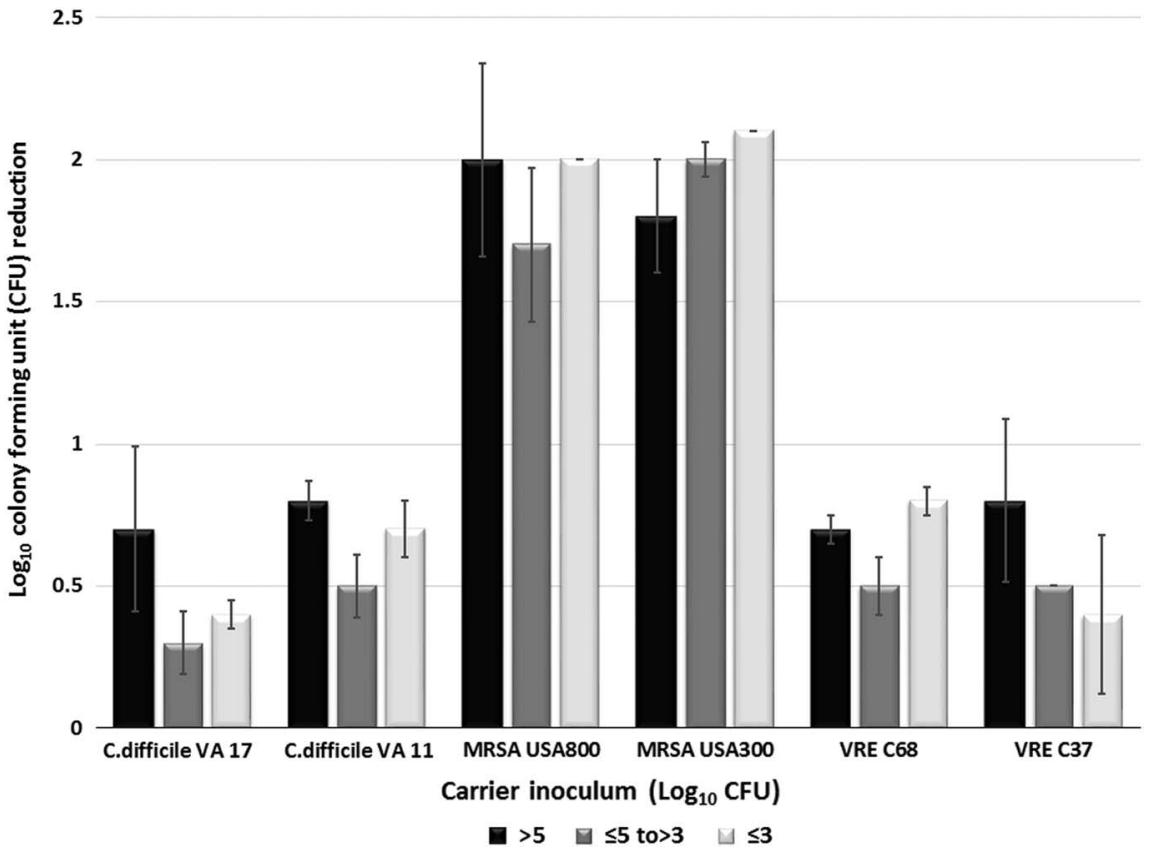

FIGURE 2. The effect of pathogen concentration on the efficacy of the pulsed xenon ultraviolet (PX-UV) device.

The $\log _{10} \mathrm{CFU}$ reduction/ $\mathrm{cm}^{2}$ of 2 strains each of Clostridium difficile spores, methicillin-resistant Staphylococcus aureus (MRSA), and vancomycin-resistant Enterococcus (VRE) inoculated onto carriers is shown. Carriers contained either $>5$, $\leq 5$ and $>3$, or $\leq 3 \log _{10} \mathrm{CFU}$ of each pathogen. The carriers were irradiated for 10 minutes at a distance of 4 feet from the PX-UV device. The means of the data from experiments conducted in triplicate are presented. Error bars indicate standard error.

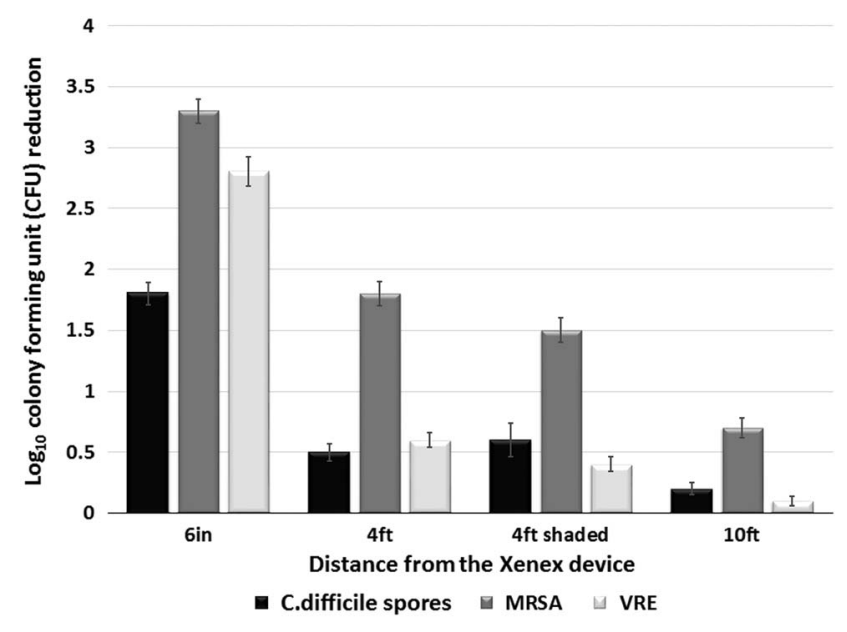

FIGURE 3. The effect of distance on the efficacy of the pulsed xenon ultraviolet (PX-UV) device.

The $\log _{10} \mathrm{CFU}$ reduction $/ \mathrm{cm}^{2}$ of Clostridium difficile spores, methicillinresistant Staphylococcus aureus (MRSA), and vancomycin-resistant Enterococcus (VRE) at increasing distances and shaded from the direct field of radiation delivered by the PX-UV device is shown. Carriers contained $5 \log _{10} \mathrm{CFU}$ of each pathogen. The carriers were irradiated for 10 minutes at a distance of 6 in, 4 feet, 4 feet shaded, and 10 $\mathrm{ft}$ from the PX-UV device. The means of the data from experiments conducted in triplicate are presented. Error bars indicate standard error. surfaces in hospital rooms with a 10-minute exposure time. Increasing the distance from the device dramatically reduced the killing efficacy of PX-UV irradiation, whereas pathogen concentration, organic load, and shading from the direct field of radiation did not. PX-UV was less effective than continuous UV-C in reducing pathogen recovery on glass slides with a 10minute exposure time in similar hospital rooms.

Our findings are consistent with previous studies demonstrating the efficacy of PX-UV disinfection for reduction of VRE, MRSA, and heterotrophic bacteria from surfaces in healthcare facilities. ${ }^{8,11}$ In addition, our study provides 2 assessments not included in previous publications on PX-UV (ie, log reductions achieved by PX-UV on carriers and a comparison with continuous UV). Although the log reductions achieved by PX-UV on carriers at 10 minutes were relatively modest, this exposure time was sufficient to reduce contamination on real-world surfaces. We have previously demonstrated that contaminated surfaces in hospital rooms yield relatively low concentrations $\left(<1-3 \log _{10} \mathrm{CFU}\right.$ per site sampled using swabs) of healthcare-associated pathogens. ${ }^{13,17}$ This observation is corroborated in the current study and may contribute to the efficacy of the PX-UV device in realworld settings.

The PX-UV device has some important potential advantages over other UV disinfection devices. First, unlike continuous UV-C devices, xenon flash lamps do not contain mercury. 


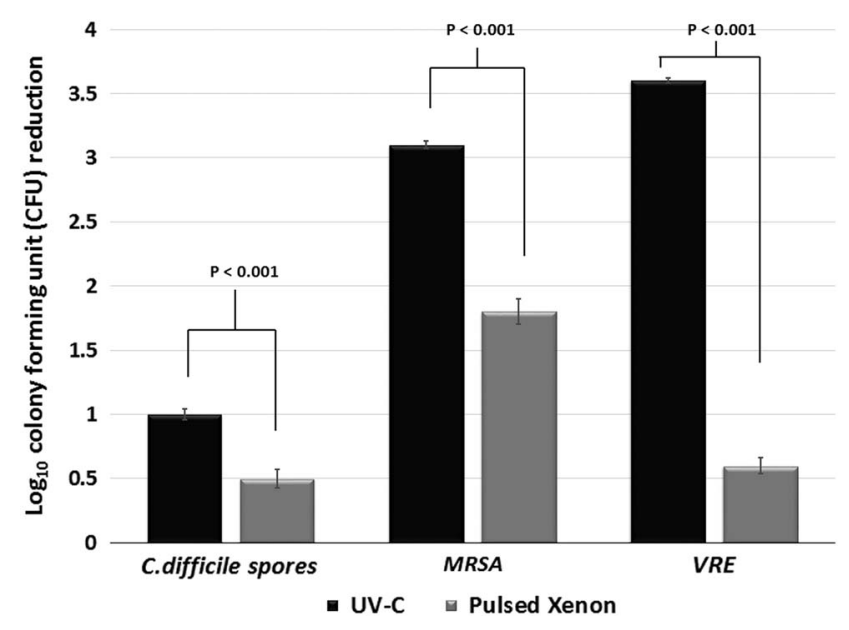

FIGURE 4. The efficacy of pulsed xenon ultraviolet (PX-UV) versus continuous mercury UV-C for killing of pathogens.

A comparison of the $\log _{10} \mathrm{CFU}$ reduction $/ \mathrm{cm}^{2}$ of Clostridium difficile spores, methicillin-resistant Staphylococcus aureus (MRSA), and vancomycin-resistant Enterococcus (VRE) by PX-UV and continuous mercury UV-C is shown. Carriers contained $5 \log _{10} \mathrm{CFU}$ of each pathogen. The carriers were irradiated for 10 minutes at a distance of 4 feet from the devices. The means of the data from experiments conducted in triplicate are presented. Error bars indicate standard error.

Therefore, there are no safety hazards associated with disposal or exposure to mercury. Second, the manufacturer recommends a relatively brief disinfection cycle (10-20 minutes per room versus up to 45 minutes for spore-killing cycles of some UV-C devices) which may facilitate greater use of the devices. However, our results suggest that continuous UV-C devices might be similarly effective or more effective than PX-UV with a 10-minute exposure time. Moreover, previous studies have demonstrated that optimal killing of $C$. difficile spores by
UV-C is likely to be achieved with longer cycle times ${ }^{13,18}$; for 2 continuous UV-C devices, reductions in $C$. difficile spores at 10,20 , and 40 minutes of exposure were $\sim 1,2$, and $3 \log _{10} \mathrm{CFU}$, respectively. ${ }^{18}$ Finally, organic load did not impact the efficacy of the PX-UV device. PX-UV has previously been shown to be more effective at penetrating organic load present in waste water than UV-C emitted by low pressure mercury bulbs. ${ }^{10}$ Thus, it is possible that the organic burden present on real-world hospital surfaces might have less impact on the killing efficacy of PX-UV than UV-C. However, we have previously demonstrated that real-world organic material collected from hospital surfaces only modestly reduced the effectiveness of continuous UV-C for killing of $C$. difficile spores. ${ }^{19}$

The PX-UV device also has some potential limitations. The efficacy of PX-UV was dramatically reduced as the distance from the device was increased. Therefore, it is recommended that commonly touched surfaces (eg, bedside table, call button, telephone) be arranged close to the device for optimal exposure to irradiation. Although the PX-UV device reduced contamination on surfaces, residual contamination was not uncommon. In contrast, technologies such as hydrogen peroxide vapor may be more effective in eliminating pathogens. ${ }^{20,21}$ Further studies are needed to determine whether the level of reduction in contamination provided by the PX-UV device is sufficient to reduce rates of infection. Two recent quasiexperimental studies have reported reductions in rates of $\mathrm{C}$. difficile infection with the use of PX-UV. ${ }^{9,22}$ Randomized trials are needed to determine whether use of PX-UV or other UV devices is effective in reducing infection rates.

Our study has some limitations. First, the use of swabs and direct plating to quantify the concentrations of bacteria is imprecise at higher concentrations. In addition, recovery and release of bacteria from swabs is less than $100 \%$ and therefore we may not have detected lower levels of bacteria on surfaces. However, methods were standardized for processing all

TA вLE 1. Clostridium difficile, Methicillin-Resistant Staphylococcus aureus (MRSA), Vancomycin-Resistant Enterococcus (VRE), and Total Heterotrophic Plate Counts (HPC) on Hospital Surfaces before and after Pulsed Xenon Ultraviolet (PX-UV) Disinfection

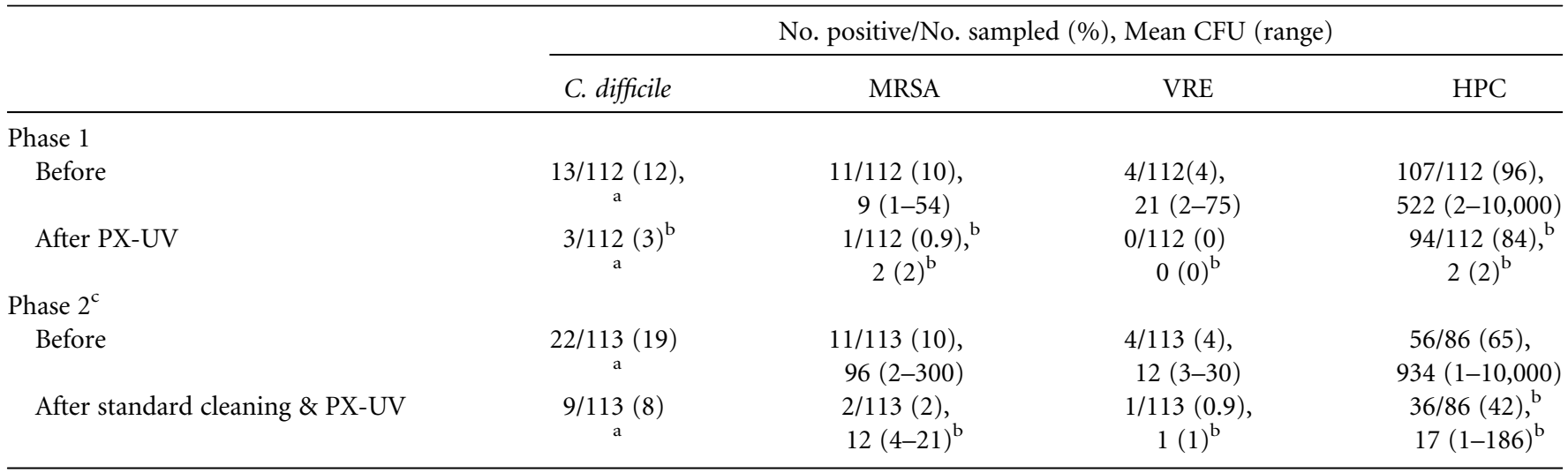

NOTE. CFU, colony-forming units.

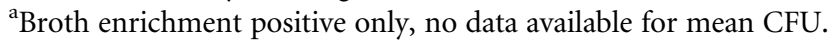

${ }^{\mathrm{b}}$ Indicates a significant reduction, $P<.01$.

${ }^{\mathrm{c}}$ In phase 2 , a total of $42 \%$ of rooms housed patients with $C$. difficile infection (in phase 1, no rooms housed such patients). 
samples so any limitations in the methodology would be equally shared by baseline and experimental groups. Second, because the PX-UV and continuous UV-C devices were housed in separate hospitals, it was not feasible to perform the comparative evaluation in the same room. We cannot completely rule out the possibility that some of the differences in results for the 2 devices were due to unappreciated differences in room characteristics. However, the experiments were performed in similar rooms with equivalent dimensions, and we found similar results for the PX-UV device when testing was done in 3 different rooms. Finally, for the evaluation of pathogen reduction in hospital rooms, we did not monitor the thoroughness of standard environmental disinfection practices.

\section{ACKNOWLEDGMENTS}

Financial support. Department of Veterans Affairs (Merit Review grant to C.J.D.) and Agency for Healthcare Research and Quality (grant 11073-H44 to C.J.D).

Potential conflicts of interest. All authors report no conflicts of interest relevant to this article.

Address correspondence to Michelle M. Nerandzic, BS, Cleveland VA Medical Center, 10701 East Blvd., Cleveland, OH 44106 (michellenerandzi@aim.com).

\section{REFERENCES}

1. Conner-Kerr TA, Sullivan PK, Gaillard J, Jones RM. The effects of ultraviolet radiation on antibiotic-resistant bacteria in vitro. Ostomy Wound Manage 1998;44:50-56.

2. Griego VM, Spence KD. Inactivation of Bacillus thuringiensis spores by ultraviolet and visible light. Appl Env Microbiol 1977;35:906-910.

3. Hercik F. Action of ultraviolet light on spores and vegetative forms of Bacillus megatherium sp. J Gen Physiol 1936;20:589-594.

4. Setlow P. Spores of Bacillus subtilis: their resistance to and killing by radiation, heat and chemicals. J Appl Microbiol 2006;101:514-525.

5. Setlow P. Resistance of spores of Bacillus species to ultraviolet light. Environ Mol Mutagen 2001;38:97-104.

6. Thai TP, Keast DH, Campbell KE, Woodbury MG, Houghton PE. Effect of ultraviolet light $\mathrm{C}$ on bacterial colonization in chronic wounds. Ostomy Wound Manage 2005;51:32-45.

7. Owens MU, Deal DR, Shoemaker MO, et al. High-dose ultraviolet $\mathrm{C}$ light inactivates spores of Bacillus subtilis var. niger and Bacillus anthracis Sterne on non-reflective surfaces. Appl Biosafety 2005;10:240-247.

8. Jinadatha C, Quezada R, Huber TW, Williams JB, Zeber JE, Copeland LA. Evaluation of a pulsed-xenon ultraviolet room disinfection device for impact on contamination levels of methicillinresistant Staphylococcus aureus. BMC Infect Dis 2014;14:187.
9. Levin J, Riley LS, Parrish C, English D, Ahn S. The effect of portable pulsed xenon ultraviolet light after terminal cleaning on hospital-associated Clostridium difficile infection in a community hospital. Am J Infect Control 2013;41:746-748.

10. Otaki M, Okuda A, Tajima K, Iwasaki T, Kinoshita S, Ohgaki S. Inactivation differences of microorganisms by low pressure UV and pulsed xenon lamps. Water Sci Technol 2003; 47:185-190.

11. Stibich M, Stachowiak J, Tanner B, et al. Evaluation of a pulsedxenon ultraviolet room disinfection device for impact on hospital operations and microbial reduction. Infect Control Hosp Epidemiol 2011;32:286-288.

12. Umezawa K, Asai S, Inokuchi S, Miyachi H. A comparative study of the bactericidal activity and daily disinfection housekeeping surfaces by a new portable pulsed UV radiation device. Curr Microbiol 2012;64:581-587.

13. Nerandzic MM, Cadnum JL, Pultz MJ, Donskey CJ. Evaluation of an automated ultraviolet radiation device for decontamination of Clostridium difficile and other healthcare-associated pathogens in hospital rooms. BMC Infect Dis 2010;10:197.

14. Sorg JA, Sonenshein AL. Bile salts and glycine as cogerminants for Clostridium difficile spores. J Bacteriol 2008;190(7):2505-2512.

15. Nerandzic MM, Donskey CJ. Effective and reduced-cost modified selective medium for isolation of Clostridium difficile. J Clin Microbiol 2009;47(2):397-400.

16. National Committee for Clinical Laboratory Standards. Methods for dilution antimicrobial susceptibility tests for bacteria that grow aerobically; approved standard, M7. Wayne, PA: Clinical and Laboratory Institute, 2005.

17. Nerandzic MM, Cadnum JL, Eckart KE, Donskey CJ. Evaluation of a hand-held far-ultraviolet radiation device for decontamination of Clostridium difficile and other healthcare-associated pathogens. BMC Infect Dis 2012;12:120.

18. Nerandzic MM, Fisher CW, Donskey CJ. Sorting through the wealth of options: Comparative evaluation of 2 ultraviolet disinfection systems. PLOS ONE, 9:e107444.

19. Zhang A, Nerandzic MM, Kundrapu S, Donskey CJ. Does organic material on hospital surfaces reduce the effectiveness of hypochlorite and UV radiation for disinfection of Clostridium difficile? Infect Control Hosp Epidemiol 2013;34:1106-1108.

20. Barbut F, Menuet D, Verachten M, Girou E. Comparison of the efficacy of a hydrogen peroxide dry-mist disinfection system and sodium hypochlorite solution for reduction of Clostridium difficile spores. Infect Control Hosp Epidemiol 2009;30:507-514.

21. Boyce JM, Havill NL, Otter JA, et al. Impact of hydrogen peroxide vapor room decontamination on Clostridium difficile environmental contamination and transmission in a healthcare setting. Infect Control Hosp Epidemiol 2008;29:723-729.

22. Haas JP, Menz J, Dusza S, Montecalvo MA. Implementation and impact of ultraviolet environmental disinfection in an acute care setting. Am J Infect Control 2014;42:586-590. 